\title{
Physiological Effects of GLT1 Modulation in Saccharomyces cerevisiae Strains Growing on Different Nitrogen Sources
}

\author{
Marco Brambilla ${ }^{1 \dagger}$, Giusy Manuela Adamo ${ }^{1 \dagger}$, Gianni Frascotti ${ }^{1}$, Danilo Porro ${ }^{1,2 *}$, and Paola Branduardi ${ }^{1}$ \\ ${ }^{1}$ Department of Biotechnology and Biosciences, University of Milano-Bicocca, Piazza della Scienza 2 - 20126 Milan, Italy \\ ${ }^{2} \mathrm{SYSBIO}$ - Centre of Systems Biology, Milano and Roma, Italy
}

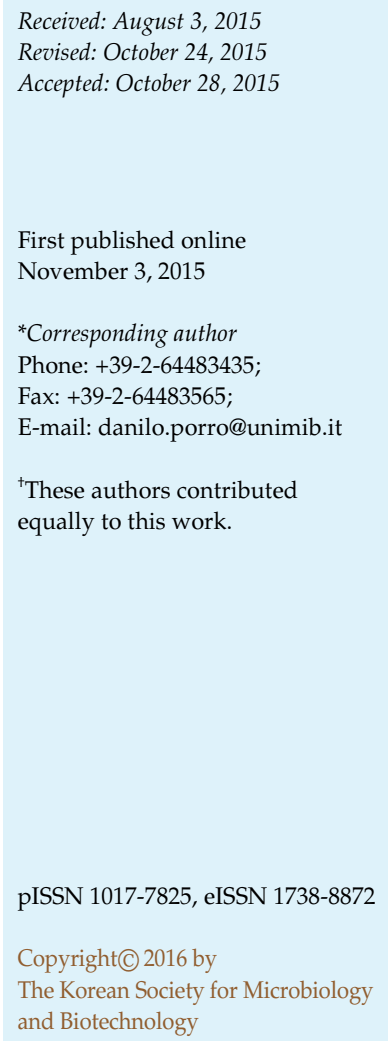

Saccharomyces cerevisiae is one of the most employed cell factories for the production of bioproducts. Although monomeric hexose sugars constitute the preferential carbon source, this yeast can grow on a wide variety of nitrogen sources that are catabolized through central nitrogen metabolism (CNM). To evaluate the effects of internal perturbations on nitrogen utilization, we characterized strains deleted or overexpressed in GLT1, encoding for one of the key enzymes of the CNM node, the glutamate synthase. These strains, together with the parental strain as control, have been cultivated in minimal medium formulated with ammonium sulfate, glutamate, or glutamine as nitrogen source. Growth kinetics, together with the determination of protein content, viability, and reactive oxygen species (ROS) accumulation at the single cell level, revealed that GLT1 modulations do not significantly influence the cellular physiology, whereas the nitrogen source does. As important exceptions, GLT1 deletion negatively affected the scavenging activity of glutamate against ROS accumulation, when cells were treated with $\mathrm{H}_{2} \mathrm{O}_{2}$, whereas Glt1p overproduction led to lower viability in glutamine medium. Overall, this confirms the robustness of the CNM node against internal perturbations, but, at the same time, highlights its plasticity in respect to the environment. Considering that side-stream protein-rich waste materials are emerging as substrates to be used in an integrated biorefinery, these results underline the importance of preliminarily evaluating the best nitrogen source not only for media formulation, but also for the overall economics of the process.

Keywords: Central nitrogen metabolism, GLT1, Saccharomyces cerevisiae, glutamate, glutamine, ammonium sulfate

\section{Introduction}

Natural and engineered yeast cell factories are today extensively used for commercial productions [16]. Based upon their innate metabolic abilities, yeasts have been employed since several decades for large-scale production of different natural compounds. In this respect, there are more than 600 yeast factories in operation in the world [4]. Furthermore, with the advent of recombinant DNA technology, it has become possible to introduce traits for the production of desired non-natural compounds: heterologous proteins and metabolites [30]. The scientific and technological platforms leading to the production of recombinant proteins seem under consolidation, with the exception of membrane proteins, with Escherichia coli and Saccharomyces cerevisiae being the two microbial workhorses mainly exploited for products under commercialization. Conversely, the production of heterologous and endogenous metabolites by engineered cell factories always and strongly suffers from extensive regulation of cellular metabolism, which easily evolves to ensure robustness. Indeed, the main research effort today is probably related to the design of robust and stable strains to match the limiting conditions often occurring during industrial fermentation. In this respect, central carbon metabolism (CCM), central nitrogen metabolism (CNM) and energy metabolism (EM, which 


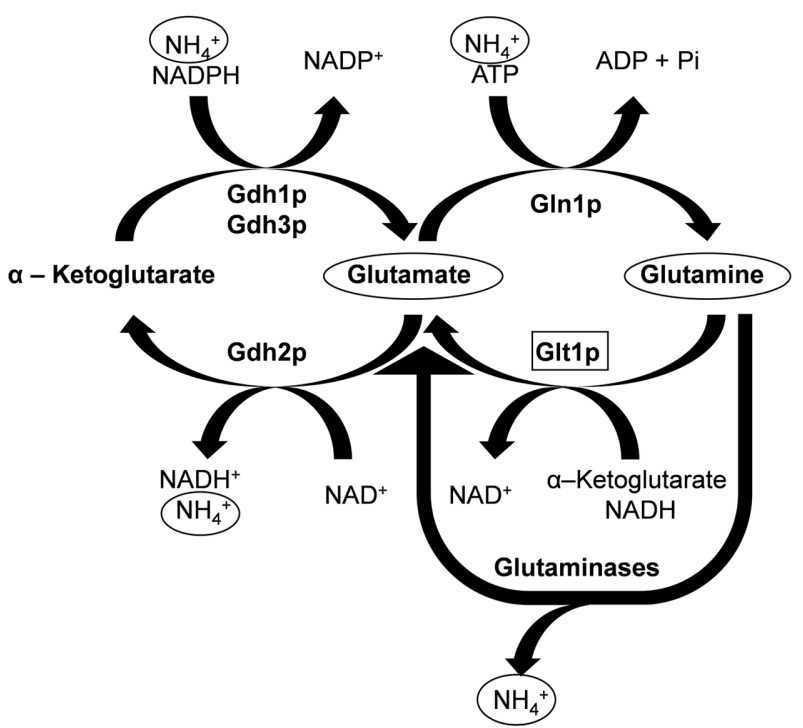

Fig. 1. Representation of central nitrogen metabolism in $S$. cerevisiae (redrawn from [23]).

includes redox metabolism), including their interconnections, play a crucial role for every microbial process and are emerging targets for eliciting profound cellular rewiring (see, as example, [11]).

Non-engineered $S$. cerevisiae strains are mainly addicted to monomeric hexose sugars as carbon source, glucose being the favorite one, while the spectrum of nitrogen sources is wider. CNM in S. cerevisiae $[13,21,23]$ is based on five important enzymatic reactions (Fig. 1). The first is catalyzed by glutamate dehydrogenase 1 (Gdh1p), the GDH1 gene product, which converts $\alpha$-ketoglutarate and ammonia in glutamate, oxidizing NADPH:

\footnotetext{
$\alpha$-Ketoglutarate $+\mathrm{NH}_{4}^{+}+\mathrm{NADPH}+\mathrm{H}^{+} \leftrightarrow$ Glutamate + $\mathrm{NADP}^{+}$
}

Another isoform of this enzyme is Gdh3p, encoded by $G D H 3$; its expression is negatively regulated in the presence of glucose, and is induced in the presence of ethanol [10].

The second reaction is catalyzed by glutamine synthetase (Gln1p-GS), the GLN1 gene product, which converts glutamate and ammonia in glutamine, consuming one ATP:

$$
\text { Glutamate }+\mathrm{NH}_{4}^{+}+\mathrm{ATP} \rightarrow \text { Glutamine }+\mathrm{ADP}+\mathrm{Pi}
$$

This is the sole reaction synthesizing glutamine in the cells; as a consequence, the lack of this enzymatic activity results in glutamine auxotrophic strains [23].

Glutamate dehydrogenase 2 (Gdh2p), the GDH2 gene product, catalyzes the reaction opposite to Gdh1p:
Glutamate $+\mathrm{NAD}^{+} \leftrightarrow \alpha$-Ketoglutarate $+\mathrm{NH}_{4}^{+}+\mathrm{NADH}$ $+\mathrm{H}^{+}$

When glutamate is utilized as the sole nitrogen source, cells can obtain the required ammonia to synthesize glutamine only through the reaction catalyzed by Gdh2p [23].

Glutamate synthase (Glt1p, also named as GOGAT), the GLT1 gene product, converts $\alpha$-ketoglutarate and glutamine into two molecules of glutamate, oxidizing NADH $[23,27]$ :

$\alpha$-Ketoglutarate + Glutamine $+\mathrm{NADH}+\mathrm{H}^{+} \rightarrow 2$ Glutamate $+\mathrm{NAD}^{+}$

Finally, glutaminases A and B catalyze the deamination of glutamine to glutamate and ammonium [13, 33].

In addition to regulations operating at the transcriptional level, the redox state of the cell contributes to determine the equilibrium among these reactions.

The main purpose of this work was to analyze the effects of modulation of one of the key elements of CNM. More in detail, we analyzed glt1 4 and GLT1 overexpressing CEN.PK strains during batch-flask growth on ammonium, glutamate, or glutamine, in aerobic conditions. To the best of our knowledge, this is the first description of the physiology of $S$. cerevisiae strains that exclusively modulate GLT1 expression.

\section{Materials and Methods}

\section{S. cerevisiae Strains Construction}

All the primers and $S$. cerevisiae strains employed and developed in this study are listed in Tables 1 and 2, respectively.

The CEN.PK $C$ control strain was obtained by transforming the reference strain CEN.PK 102-5B with the integrative plasmids pYX012, pYX022, and pYX042 (from R\&D System, Wiesbaden, Germany).

The strain CEN.PK glt1 $\Delta$ was constructed by deleting the first 1,650 bp of gene GLT1. First the KanMX4 cassette was amplified from the pFA6-a-KanMX4 [37] plasmid with the primers dGLT1 KAN FW and dGLT1 KAN REV2. Then the reference strain CEN.PK 102-5B was transformed and selected on YPD plates with $200 \mathrm{mg} / \mathrm{l}$ of G418. Deletion was verified by PCR with the pairs of primers dGLT1 CNTR FW 2 / KAN RV and KAN FW / dGLT1 CNTR RV 2, respectively.

The GLT1 overexpressing strain was created as described: the first 1,191 bp of the GLT1 genomic ORF was amplified by PCR, including the ATG starting codon, with primers GLT1_SMAI_FW and GLT1_SMAI_REV. Then, both the PCR product and the pYX042-ATG plasmid (R\&D System) were XmaI digested and a ligation reaction was performed, resulting in plasmid pYX042ATG [TPI-GLT1]. This plasmid was BglII digested inside the insert 
Table 1. List of primers used in this work.

\begin{tabular}{ll}
\hline \multicolumn{1}{c}{ Name } & \multicolumn{1}{c}{ Sequence } \\
\hline dGLT1 KAN FW & ATGCCAGTGTTGAAATCAGACAATTTCGATCCATTGGAAGCGGATCCCCGGGTTAATTAA \\
dGLT1 KAN REV2 & GGTCACTTTGAACTTTCAAAGACAAATGAATCCTCTGATATGAATTCGAAGCTCGTTTAAACTG \\
dGLT1 CNTR FW 2 & TAGAAAAGAAAGCATGCCAGT \\
KAN RV & TTAGAAAAACTCATCGAGCATCAAATG \\
KAN FW & ATGGGTAAGGAAAAGACTCACGTT \\
dGLT1 CNTR RV 2 & GCAATAGACGTGGGTCACTTTGA \\
GLT1_SMAI_FW & GCGCCGCCCGGG-ATGCCAGTGTGAAATCAGAC \\
GLT1_SMAI_REV & GCATATCCCGGG-GCTGGAACCATCCCAAGGTTCC \\
TPICNTR_FW & GATCTACGTATGGTCAT \\
GLT1CNTR_REV & GTCATCACTAGTGATGT \\
GLT1-GFP FW & ACGTGATTACAAACTATTGAAAGAATTAGCTAGTCAAGTCCGGATCCCCGGGTTAATTAA \\
GLT1-GFP RV & ATAATATACGATCATAAAATAAATAATAACTCAAGCTTTGAATTCGAGCTCGTTTAAACTGG \\
GFP RW & ATATTATTTGTATAGTTCATCCATGC \\
D GLT1 cntr fw2 & TAGAAAAGAAAGCATGCCAGTGTTA \\
\hline
\end{tabular}

Bold: sequence annealing to the genome. Italics: sequence annealing to the plasmid. Bold and italics (underlined): XmaI restriction site.

Table 2. List of strains used in this work.

\begin{tabular}{|c|c|c|}
\hline Strains & Relevant genotype & Source \\
\hline CEN.PK 102-5B & MATa, ura3-52, his3-11, leu2-3/112, TRP1, MAL2-8C, SUC2 (reference strain) & a \\
\hline CEN.PK C & MATa, ura3-52, his3-11, leu2-3/112, TRP1, MAL2-8C, SUC2, [pYX012; $p Y X 022 ; p Y X 042]$ & b \\
\hline CEN.PK glt1 $\Delta$ & MATa, ura3-52, his3-11, leu2-3/112, TRP1, MAL2-8C, SUC2, glt1::kanMX4 [pYX012; YX022; pYX042] & b \\
\hline CEN.PK TPI-GLT1 & MATa, ura3-52, his3-11, leu2-3/112, TRP1, MAL2-8C, SUC2, GLT1::pYX042[TPI-GLT1], [pYX012; pYX022] & b \\
\hline CEN.PK GLT1-GFP & MATa, ura3-52, his3-11, leu2-3/112, TRP1, MAL2-8C, SUC2, GLT1::GLT1-GFP, [pYX012; $p Y X 042]$ & b \\
\hline CEN.PK TPI-GLT1-GFP & $\begin{array}{l}\text { MATa, ura3-52, his3-11, leu2-3/112, TRP1, MAL2-8C, SUC2, GLT1::pYX042[TPI-GLT1], } \\
\text { GLT1::GLT1-GFP, [pYX012] }\end{array}$ & $\mathrm{b}$ \\
\hline
\end{tabular}

${ }^{a}$ Kindly provided by Dr. P. Kotter (Institute of Microbiology, Johann Wolfang Goethe-University, Frankfurt, Germany).

${ }^{\mathrm{b}}$ This study.

and, once linearized, the reference strain CEN.PK 102-5B was transformed with it. The proper recombination in the GLT1 locus was verified by PCR with primers TPICNTR_FW and GLT1CNTR_REV.

The strains CEN.PK GLT1-GFP and CEN.PK TPI-GLT1-GFP were created as follows: a fragment containing the genes GFP and HIS3 (separated by a linker) was amplified by PCR from pFA6aGFP(S65T)-His3MX6 with the primers GLT1-GFP FW and GLT1GFP RV. With this construct, strains CEN.PK 102-5B and CEN.PK TPI-GLT1 were transformed and selected on appropriate plates without histidine. Analytical PCR with primers GFP RW and D GLT1 cntr fw2 was performed on transformants to verify proper recombination.

When necessary, all the strains of this study were complemented to obtain the corresponding prototrophic strain with the integrative plasmids previously indicated.

All the transformations were performed according to the LiAc/
PEG/ss-DNA protocol [12].

\section{Media and Growth Kinetics}

Yeast cultures were shake-flask grown in modified Verduyn medium [36] composed of $150 \mathrm{~g} / 1$ glucose, vitamins and traces $(1,000 \times)$, water, and saline solution at $\mathrm{pH} 5$. The latter were prepared with $0.5 \mathrm{~g} / 1 \mathrm{MgSO}_{4} \cdot 7 \mathrm{H}_{2} \mathrm{O}$ and $3 \mathrm{~g} / 1 \mathrm{KH}_{2} \mathrm{PO}_{4}$, and subsequently supplied with different nitrogen sources as follows: $15 \mathrm{~g} / 1\left(\mathrm{NH}_{4}\right)_{2} \mathrm{SO}_{4}$ or $48.96 \mathrm{~g} / 1$ glutamate or $18 \mathrm{~g} / 1$ glutamine (corresponding to $30.6 \mathrm{mM}$ of ammonium released); $2.5 \mathrm{~g} / 1$ $\left(\mathrm{NH}_{4}\right)_{2} \mathrm{SO}_{4}$ or $8.16 \mathrm{~g} / 1$ glutamate or $3 \mathrm{~g} / 1$ glutamine (corresponding to $5.1 \mathrm{mM}$ of ammonium released). Pre-cultures were performed in minimal medium with $20 \mathrm{~g} / 1$ glucose and $6.7 \mathrm{~g} / 1$ yeast nitrogen base. Cells were inoculated at the starting optical density $\left(\mathrm{OD}_{660 \mathrm{~nm}}\right)$ of 0.05 in $250 \mathrm{ml}$ flasks containing $50 \mathrm{ml}$ of medium. Fermentations were performed at $30^{\circ} \mathrm{C}$ under continuous shaking (160 rpm) and cellular growth was followed by measuring the $\mathrm{OD}_{660 \mathrm{~nm}}$ using the 
spectrophotometer UV-1601 (Shimadzu).

\section{CFU Analysis}

Cell viability was determined as follows: cells at 0.5 OD were serial-diluted 1,000 times and then $200 \mu \mathrm{l}$ was plated in triplicates on YPD agar. Colony-forming units (CFU) were measured after 2 days of growth at $30^{\circ} \mathrm{C}$.

\section{Preparation of Soluble and Organelle-Associated Protein Extract}

Cells in exponential phase of growth were washed twice with cold deionized water and resuspended in extraction buffer composed of $0.1 \mathrm{M}$ potassium phosphate buffer ( $\mathrm{pH} 7.5), 1 \mathrm{mM}$ ethylenediaminetetraacetic acid (EDTA), $1 \mathrm{mM}$ dithiothreitol (DTT), $1 \mathrm{mM}$ protease inhibitor cocktail, and $1 \mathrm{mM}$ phenylmethanesulfonylfluoride (PMSF). The cell suspension was subjected to three cycles of mechanical disruption with the FastPrep-24 (MP Biomedical). Cellular lysate was first centrifuged at $700 \times g$ for $10 \mathrm{~min}$ at $4^{\circ} \mathrm{C}$ to separate supernatants from glass beads and cellular debris and then at $20,817 \times \mathrm{g}$ for $20 \mathrm{~min}$ at $4^{\circ} \mathrm{C}$ to obtain the soluble protein fraction; the organelle-associated protein fraction in the pellet was solubilized by the detergent DS1 composed of $7 \mathrm{M}$ urea, $2 \mathrm{M}$ thiourea, $40 \mathrm{~g} / 1$ 3-[(3-cholamidopropyl)dimethylammonium]1-propanesulfonate (CHAPS), $60 \mathrm{mM} \mathrm{DTT}$, and $20 \mathrm{mM}$ 2-iodoacetamide (IAA). Protein concentration was estimated according to Bradford [3], using bovine serum albumin as the reference.

\section{Enzymatic Assays}

Glutamate synthase enzymatic assay was performed as previously described [7], slightly modified as follows: the reaction mixture (1 ml final volume) contained buffer phosphate $50 \mathrm{mM}(\mathrm{pH} \mathrm{7)}$, NADH $10 \mathrm{mM}, \alpha$-ketoglutarate $50 \mathrm{mM}$, and the sample. To start the reaction, glutamine $100 \mathrm{mM}$ was added. In parallel, this assay was repeated with azaserine $5 \mathrm{mM}$, a competitive inhibitor of Glt1p used as a control. NADPH glutamate dehydrogenase 1 and $\mathrm{NADH}$ glutamate dehydrogenase 2 activities were determined as previously described [15], slightly modified as follows: the reaction mixture ( $1 \mathrm{ml}$ final volume) contained buffer phosphate $50 \mathrm{mM}$ (pH 7), NADPH or NADH $10 \mathrm{mM}, \alpha$-ketoglutarate $50 \mathrm{mM}$, and the sample. To start the reaction, ammonium chloride $100 \mathrm{mM}$ was added. For all the assays, NADH (or NADPH) oxidation was monitored by following the decrease in absorbance at $340 \mathrm{~nm}$ for $10 \mathrm{~min}$. The $\Delta \mathrm{OD} / \mathrm{min}$ was obtained using the maximum linear rate of the reaction. One unit $(\mathrm{U})$ was defined as the amount of enzyme that oxidized $1 \mathrm{nmol}$ of NADH (or NADPH) in one minute. Glutamine synthetase activity was determined according to Kingdon et al. [20].

\section{Flow Cytometry Analysis}

Experiments were carried out with the Beckman Coulter CYTOMICS-FC 500. For propidium iodide (PI) staining, an amount of cells corresponding to $0.2 \mathrm{OD}$ was washed with $1 \times \mathrm{PBS}$ and then with Tris- $\mathrm{HCl} 50 \mathrm{mM} / \mathrm{MgCl}_{2} 15 \mathrm{mM}$ (pH 7.7) buffer. Subsequently, cells were resuspended in $1 \mathrm{ml}$ of PI $0.23 \mathrm{mM}$ (dissolved in Tris- $\mathrm{HCl} 50 \mathrm{mM} / \mathrm{MgCl}_{2} 15 \mathrm{mM}, \mathrm{pH}$ 7.7). After $20 \mathrm{~min}$ of incubation on ice and in the dark, samples were analyzed with excitation wavelength at $535 \mathrm{~nm}$ and emission wavelength at $617 \mathrm{~nm}$.

For DHR123 (dihydrorhodamine 123) and PI double-staining experiments, samples were prepared as previously described [5] but modified as follows: cells at 0.2 OD were washed and resuspended in $1 \mathrm{ml}$ of $1 \times \mathrm{PBS}$. Samples were incubated at $30^{\circ} \mathrm{C}$, in the dark, at $160 \mathrm{rpm}$ for $2 \mathrm{~h}$ in the presence of $5 \mu \mathrm{g} / \mathrm{ml}$ of DHR123 and $2 \mathrm{mM}$ of hydrogen peroxide. Cells were washed with $1 \times$ PBS, then with buffer Tris- $\mathrm{HCl} 50 \mathrm{mM} / \mathrm{MgCl}_{2} 15 \mathrm{mM}$ (pH 7.7), and finally resuspended in $1 \mathrm{ml}$ of $5 \times$ PI. Samples were analyzed with excitation wavelength at $535 \mathrm{~nm}$ for PI and $500 \mathrm{~nm}$ for DHR123 and emission wavelength at $617 \mathrm{~nm}$ and $536 \mathrm{~nm}$, respectively.

For FITC (fluorescein isothiocyanate) experiments, cells at 2 OD were resuspended in $1 \mathrm{ml}$ of cold ethanol $70 \%(\mathrm{v} / \mathrm{v})$, and incubated for $15 \mathrm{~min}$ at $-20^{\circ} \mathrm{C}$ first and then for $20 \mathrm{~min}$ at $4^{\circ} \mathrm{C}$. Then $100 \mu$ of the cellular suspension was washed once with $1 \times$ PBS, resuspended in a FITC solution $5 \mathrm{ng} / \mathrm{ml}$ (dissolved in $\mathrm{NaHCO}_{3} 50 \mu \mathrm{g} / \mathrm{ml}$ ), and incubated in the dark on ice for $30 \mathrm{~min}$. Samples were analyzed with excitation wavelength at $495 \mathrm{~nm}$ and emission wavelength at $519 \mathrm{~nm}$.

The data obtained from all the experiments were analyzed with the software Cyflogic ver. 1.2.1.

\section{Fluorescence Microscopy}

Culture samples of strains CEN.PK GLT1-GFP and CEN.PK TPI-GLT1-GFP, corresponding to $0.5 \mathrm{OD}$, were washed once with $1 \times$ PBS, and then resuspended in $30 \mu \mathrm{l}$ of $1 \times$ PBS and observed under the fluorescence microscope Nikon ECLIPSE 90i (Nikon), using the 100x objective. Images were acquired using CoolSnap CCD camera and then analyzed with the software Metamorph 6.3.

\section{Results}

Growth of Wild-Type, glt1 $\triangle$, and GLT1 Overexpressing Strains on Media Containing $\left(\mathrm{NH}_{4}\right)_{2} \mathrm{SO}_{4}$, Glutamate, or Glutamine

To characterize the effects of internal and external modulations in one of the key enzyme (glutamate synthase, encoded by GLT1) of the CNM on the growth properties of S. cerevisiae, we grew the wild type CEN.PK C, the CEN.PK glt1D, and the CEN.PK TPI-GLT1 strains in flask-batch culture on $\left(\mathrm{NH}_{4}\right)_{2} \mathrm{SO}_{4}$, glutamate, or glutamine as the nitrogen source. The nitrogen source was supplied at two different sets of concentrations, normalized to release 5.1 or $30.6 \mathrm{mM}$ of $\mathrm{NH}_{4}{ }^{+}$(see Materials and Methods), to evaluate the effects of a low and a very high nitrogen amount on cellular growth; in particular, $30.6 \mathrm{mM}$ is the highest possible concentration based on maximum glutamine 


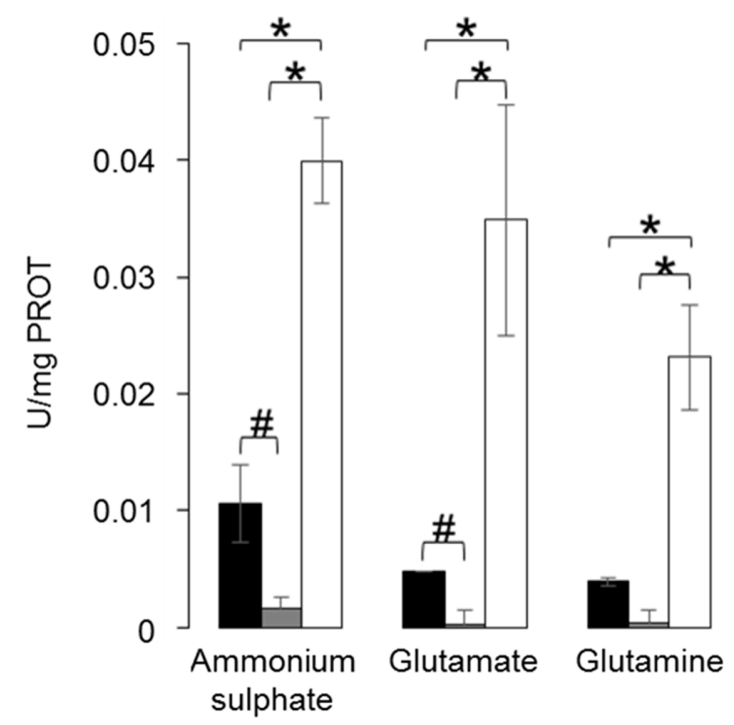

Fig. 2. Glutamate synthase activity tested on the soluble protein fraction of CEN.PK C (black columns), CEN.PK glt1 (grey columns), and CEN.PK TPI-GLT1 (white columns).

Values represent the average and the standard deviation of three independent experiments $\left({ }^{*} p \leq 0.01\right.$; $\# p \leq 0.05$, Student's $t$-test).

solubility. Glucose was always supplied at $150 \mathrm{~g} / \mathrm{l}$, reported as the maximum concentration beyond which growth inhibition occurs [26]. A high glucose concentration was selected since this is the condition very often applied when yeasts are employed as cell factories for the industrial production of biobased chemicals.

As initial control, we determined the activity of Glt1p for cells in exponential phase of growth in aerobic conditions. Since Glt1p localization is still dubious [25], the enzymatic assay was performed on both soluble and organelle- associated protein fractions, but the activity was detectable only in the first one. Data are summarized in Fig. 2. In agreement with literature data [35], the activity appeared only slightly down-regulated for wild-type strains growing in glutamate and glutamine, when compared with cells growing on ammonium (black columns). GLT1 overexpression was functional, as confirmed by fluorescence microscope observation of CEN.PK GLT1-GFP and CEN.PK TPI-GLT1GFP (data not shown) and by the higher measured activity (Fig. 2, white columns), but with no significant differences among the different nitrogen sources.

Notably, both the deletion and the overexpression of the GLT1 gene did not determine remarkable differences on the growth profiles (data not shown). The observed differences are essentially ascribable to the different nitrogen source. As a representative example, Fig. 3 shows the growth kinetics of the wild-type CEN.PK $C$ strain during aerobic growth on low (Panel A) or high (Panel B) nitrogen concentration. Yeast cells reached the highest optical density in the presence of high quantities of glutamine, whereas the lowest OD was obtained in the presence of ammonium sulfate. Moreover, when glutamate was supplemented, cells showed different trends of growth depending on the amino acid concentration, growing significantly faster when it was supplied at the lower concentration (Panel A versus Panel B).

Forward Scatter and Protein Content of Wild-Type, glt1 $\Delta$, and GLT1 Overexpressing Strains during Growth on $\left(\mathrm{NH}_{4}\right)_{2} \mathrm{SO}_{4}$, Glutamate, or Glutamine

For all the experiments described in the rest of the paper, the wild-type CEN.PK C, the CEN.PK glt1 $\Delta$, and the CEN.PK TPI-GLT1 strains were grown in aerobic conditions
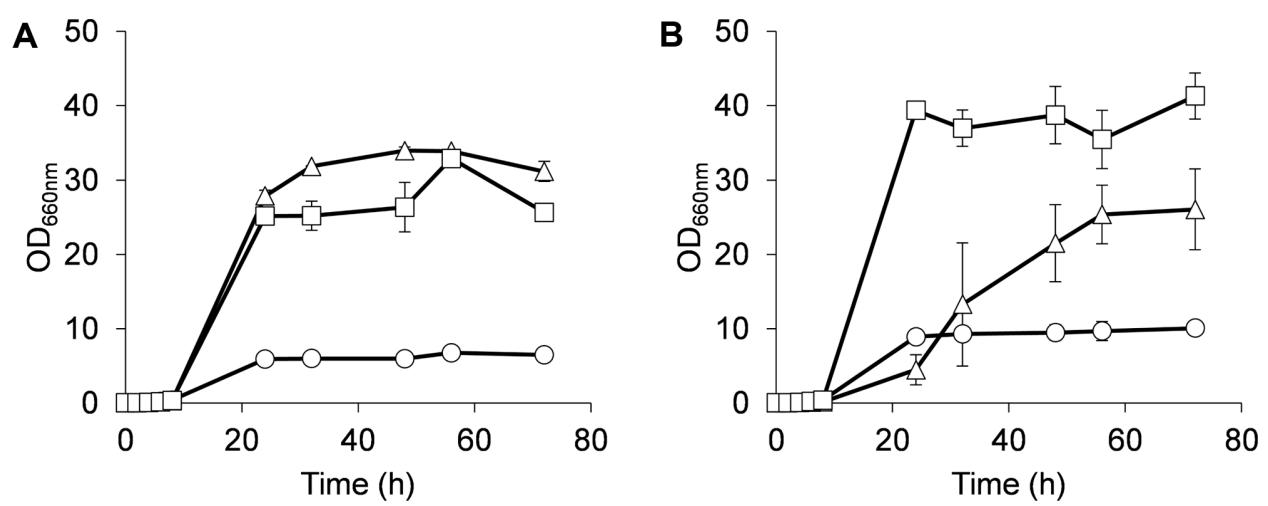

Fig. 3. Growth curve of CEN.PK C strain (representative also for the GLT1-deleted and -overexpressing strains) cultivated in aerobic conditions and supplemented with ammonium sulfate (circle), glutamate (triangle), or glutamine (square), which were supplied to release (A) $5.1 \mathrm{mM}$ or (B) $30.6 \mathrm{mM}$ of ammonium.

Results are the average and the standard deviation of three independent experiments. 

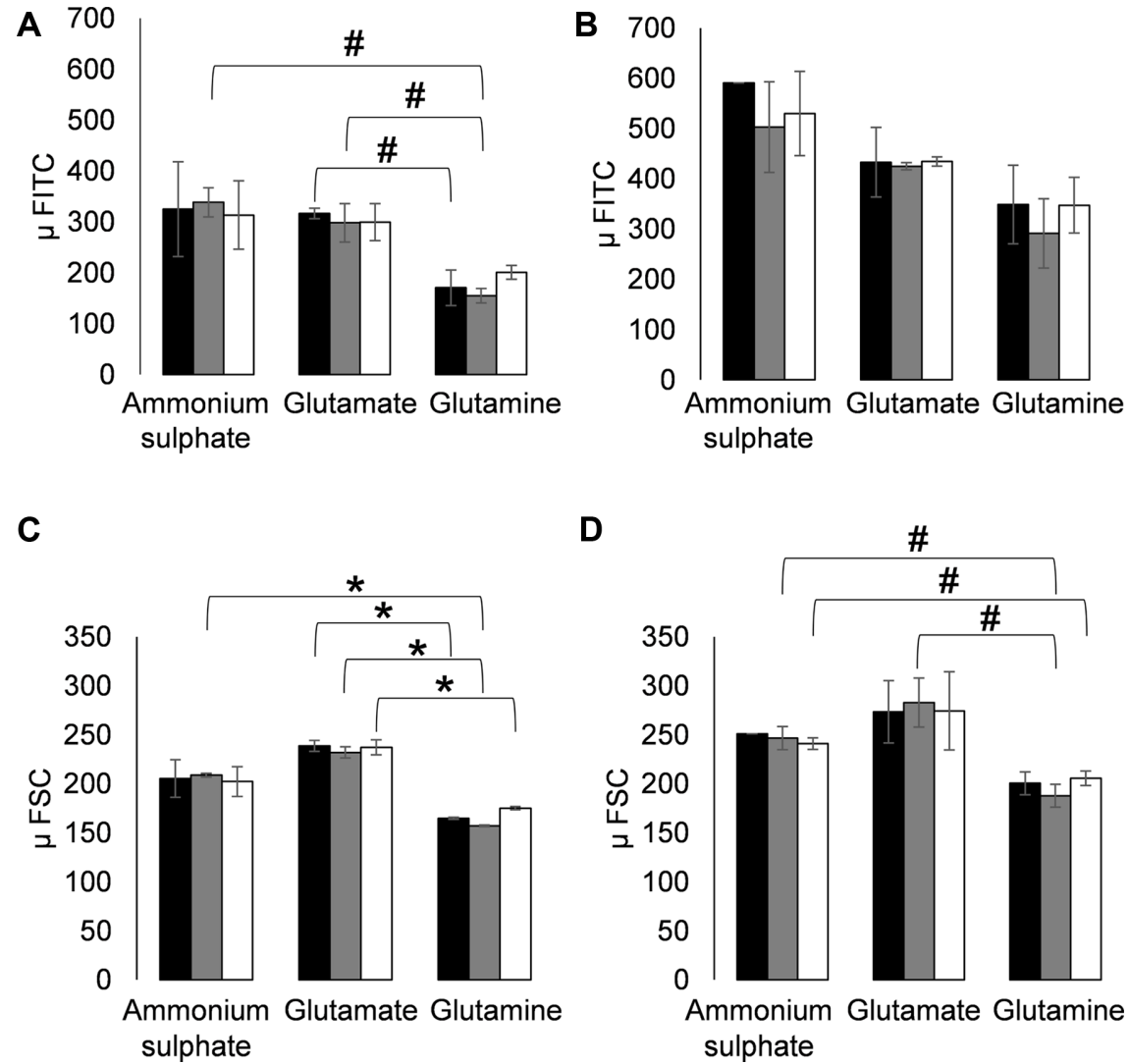

Fig. 4. Protein content evaluated in the (A) exponential or (B) stationary phase of growth, measured considering the average of FITC associated fluorescence, and average FSC evaluated in the (C) exponential or (D) stationary phase of growth. Strains shown are CEN.PK C (black columns), CEN.PK glt1ム (grey columns), and CEN.PK TPI-GLT1 (white columns). Nitrogen sources were supplied to release $30.6 \mathrm{mM}$ of ammonium.

Results represent the average and the standard deviation of three independent experiments ( ${ }^{*} p \leq 0.01$; $\# p \leq 0.05$; Student's $t$-test).

in flask-batch culture on ammonium, glutamate, and glutamine supplied to release $30.6 \mathrm{mM}$ of ammonium, as described in Fig. 3B. For cells harvested both in the exponential and stationary phases of growth, we determined the cell protein content and the cell volume at the single cell level by flow cytometry (Fig. 4). The first parameter was estimated using FITC, a typical marker of proteins [24] that binds their N-terminal amine group, while the cell volume is related to forward scatter signal (FSC). Indeed this parameter measures the light scattered when each cell passes through the laser beam, providing a measurement that is related to cell shape, cell orientation, and cell volume.

As for the data described in the previous paragraph, remarkable differences are ascribable to the different media and not to the modulation of GLT1. The lower FITC and FSC signals were registered during growth on glutamine, and the higher FSC signals for cells grown in glutamatesupplemented medium (Fig. 4). This indicates that the cell dimension is minimal when cells are growing in the presence of glutamine and maximal in the presence of glutamate, under the tested conditions. Independently from the nitrogen source, both cell sizes and cell volume contents tend to increase in the stationary phase of growth (Panels B-D).

PI and ROS Accumulation in Wild-Type, glt1 $\Delta$, and GLT1 Overexpressing Strains during Growth on $\left(\mathrm{NH}_{4}\right)_{2} \mathrm{SO}_{4}$, Glutamate, or Glutamine

The potential of the flow cytometry platform was utilized to determine the viability and reactive oxygen species (ROS) accumulation at the single cell level. Cells in the exponential and stationary phases of growth were first stained with propidium iodide, a marker for determining injured/dead cells [14]. In the exponential phase of growth, no big differences were observed among the strains or media (Fig. 5A), and the fraction of PI-positive cells was 

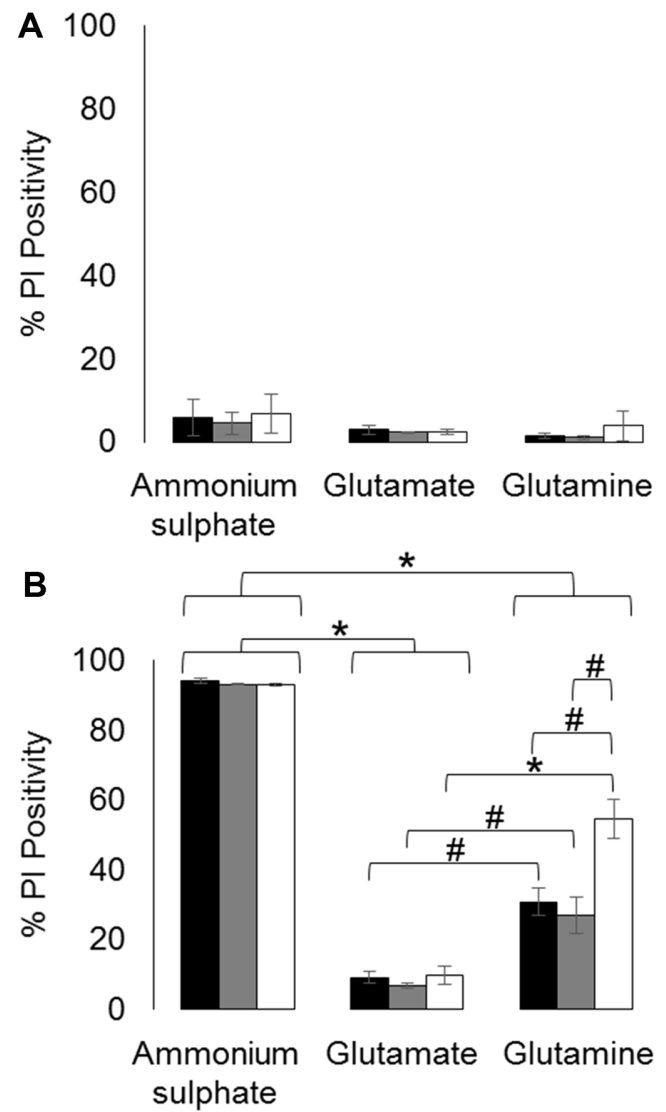

Fig. 5. PI accumulation in the (A) exponential and (B) late stationary phases of growth, measured as the percentage of PI-positive cells, for the strains CEN.PK C (black columns), CEN.PK glt1 (grey columns), and CEN.PK TPI-GLT1 (white columns).

Values represent the average and the standard deviation of three independent experiments ( ${ }^{*} p \leq 0.01$; $\# p \leq 0.05$; Student's $t$-test).

very low. Remarkably, in the very late stationary phase, positivity to PI was particularly high for cells grown in the presence of ammonium sulfate, diversely to the data obtained for cells growing in the presence of glutamate and glutamine (Fig. 5B). Notably, in the presence of glutamine, the PI signals were higher than in the presence of glutamate, with CEN.PK TPI-GLT1 being the most PIpositive strain (Fig. 5B).

Considering that significant changes in viability have been observed in the different conditions, we sought to determine whether a correlation with ROS accumulation could exist. Therefore, the three strains of this study were challenged with $\mathrm{H}_{2} \mathrm{O}_{2}$ for triggering ROS production and accumulation inside the cells that were subsequently double stained, as previously reported [5], with both PI and DHR123 (see Materials and Methods). The uncharged and non-fluorescent DHR123 passively diffuses across membranes into the cell where, in the presence of ROS, it is oxidized to cationic Rhodamine 123, its fluorescent counterpart, through the Fenton reaction [19, 22].

Fig. 6A shows the DHR123 versus PI cytogram for unchallenged cells. Each dot represents a single cell. The low DHR123 and low PI signals are indicative of a healthy growing yeast population (Panel A, circle with continuous line). Challenging the cells in the exponential phase of growth on ammonium sulfate (control - Panel B) or glutamine (Panel C) for $2 \mathrm{~h}$ with $\mathrm{H}_{2} \mathrm{O}_{2}$ resulted in a yeast population completely ROS positive (circle with double line), also independently from GLT1 modulation. Furthermore, some of the cells showed a very strong PI signals (circle with dotted line) compared with the unstressed ones. As described above, similar results have been obtained independently from the genetic background of the yeast strain (CEN.PK C, CEN.PK glt1 1 , and CEN.PK TPI-GLT1), indicating that the observed patterns are only nitrogen source dependent. Differently, challenging cells in the exponential phase of growth in glutamate medium resulted in two clear distinct subpopulations, one ROS positive and the other negative (Panels D-F). Also in this case, in each cytogram it is possible to identify some yeast cells with higher PI signals than the unstressed ones, independently from the genetic background. A comparative analysis indicated that the highest fraction of DHR123-positive cells was observed in the CEN.PK glt1 $\Delta$ strain $(\sim 40 \%$, versus $\sim 24 \%$ and $\sim 21 \%$ for the wild-type and CEN.PK TPI-GLT1 strains, respectively; Panels D-F).

Overall, flow cytometric data confirmed a pro-active role of glutamate in preventing the accumulation of ROS, probably with glutamate required for the synthesis of glutathione [28], one of the main radical scavengers in $S$. cerevisiae. Indeed, it has been shown that the supply of glutamate for glutathione biosynthesis was likely to be a factor affecting ROS accumulation and cell death in yeast [29]. Furthermore, it has to be mentioned that an involvement of the GABA pathway, starting from glutamate, has been described in the literature to be associated with scavenging properties against ROS $[6,8]$.

\section{CNM Enzymatic Assays}

The activities of the four CNM enzymes (Glt1p, Gln1p, Gdh2p, and Gdh1p) were then determined in wild-type CEN.PK $C$ and CEN.PK TPI-GLT1 strains. Gln1p, Gdh2p, and Gdh1p localization is cytosolic [25] and, therefore, only the soluble protein fraction was considered for assays. Data are summarized in Fig. 7. 


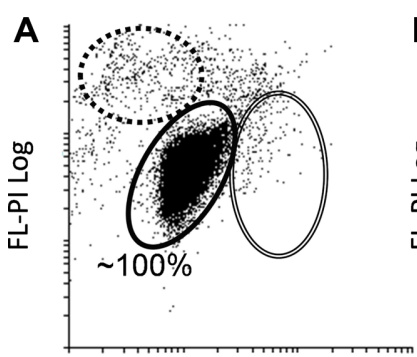

FL-DHR Log

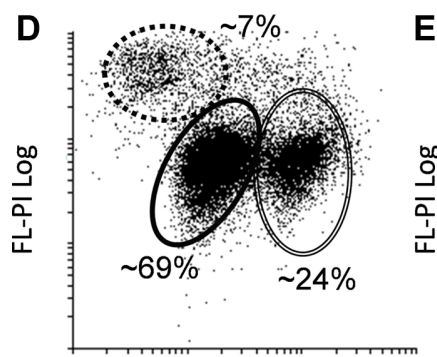

FL-DHR Log

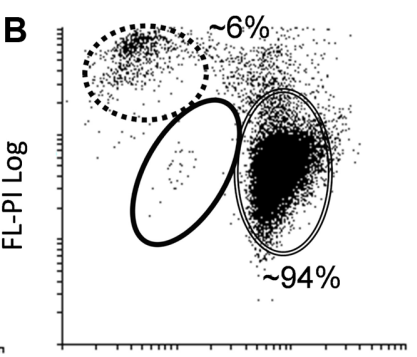

FL-DHR Log

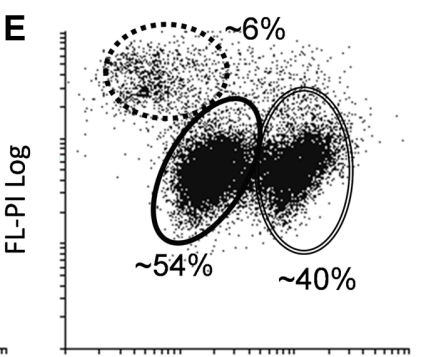

FL-DHR Log

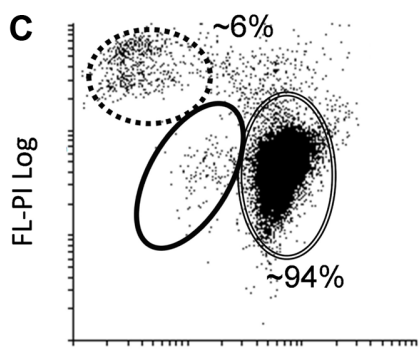

FL-DHR Log

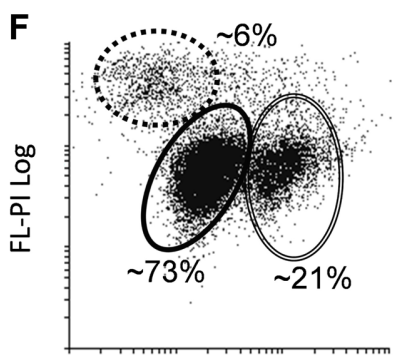

FL-DHR Log

Fig. 6. PI and cellular ROS accumulations for samples harvested in the exponential phase of growth and shocked for $2 \mathrm{~h}$ with $2 \mathrm{mM}$ of hydrogen peroxide. (A) Typical dot plot of untreated cells. (B) Typical dot plot of shocked cells in the presence of ammonium sulfate. (C) Typical dot plot of shocked cells in the presence of glutamine. (D, E, F) Dot plots of shocked CEN.PK C, CEN.PK glt1 , and CEN.PK TPI-GLT1 strains in the presence of glutamate. The circle with continuous line indicates cells in a healthy state, the circle with dotted line PI-positive cells, and the circle with double line DHR123-positive cells.

Each panels show the average percentage values of two independent experiments.

Results show that GLT1 overexpression led to an effectively higher Glt1p activity compared with the parental strain (CEN.PK C), independently from the media (Panel A). However, this perturbation does not affect the other three enzymatic activities (Panels B-D). Indeed, values tend to be similar between the two strains. Nevertheless, significant changes in activity values could be noticed comparing the three media, independently from the genetic background. Both Gln1p (Panel B) and Gdh2p (Panel C) activities were lower in glutamine medium than in glutamate one, whereas, despite present, this reduction was not statistically relevant for Gdh1p (Panel D) activity. Comparing ammonium sulfate versus glutamine, only Gln1p activity (Panel B) was significantly higher in the presence of the first nitrogen source. Finally, despite that all the activities tend to be slightly lower in the presence of ammonium sulfate than in glutamate medium, only the Gdh2p activity of the CEN.PK TPI-GLT1 strain was significantly reduced (Panel C). Trying to find a possible explanation, we speculated that GLT1 overexpression might increase glutamate levels that in turn, as a direct substrate of Gdh2p, could raise its activity. Therefore, in the presence of this amino acid, this effect might be even stronger. Furthermore, considering that the transcription factor Gln3p in the presence of glutamate can cross the nuclear membrane, stimulating CNM genes expression [23, $31,35,39]$, all the activities were generally increased in the presence of this nitrogen source. On the contrary, since glutamine is a strong activator of the TOR pathway (target of rapamycin), which indirectly prevents the expression of all the four genes $[9,21,27,39], \mathrm{CNM}$ activities (but in our case with the exception of Glt1p) tend to be very low compared with glutamate medium, significantly in the case of Gln1p and Gdh2p (Panels B and C).

\section{Discussion}

S. cerevisiae is one of the successful workhorses for industrial applications (see also Introduction). This yeast is able to metabolize a wide variety of nitrogen sources via enzymatic reactions that are indirectly linked in a network of physiological responses. These responses and their regulations are crucial to the yeast for optimizing the exploitation of the environment and are therefore crucial to improve the biotransformation of protein-rich raw materials, such as whey or exhausted biomasses derived from 
A
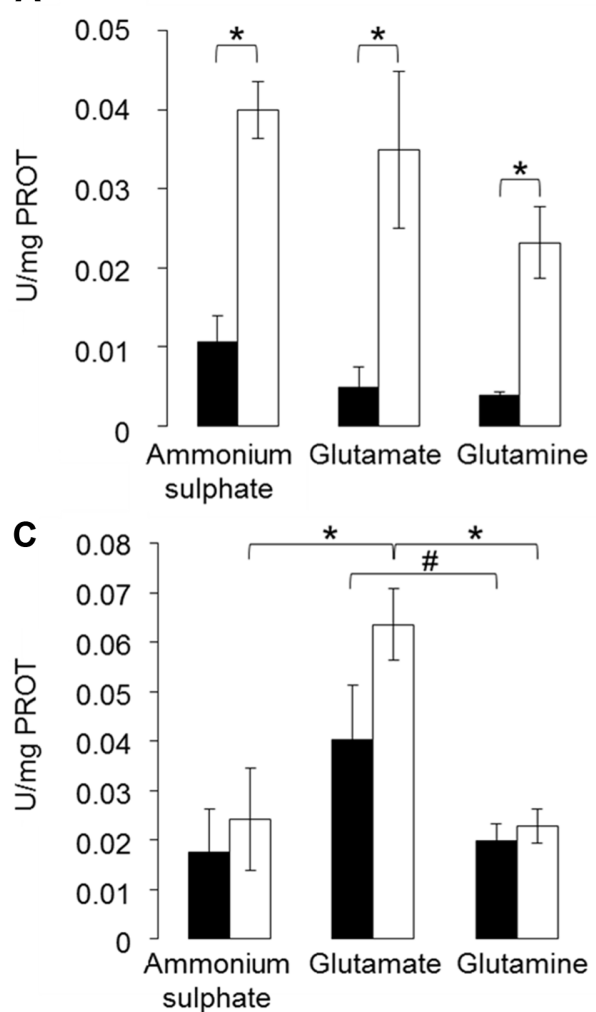

B
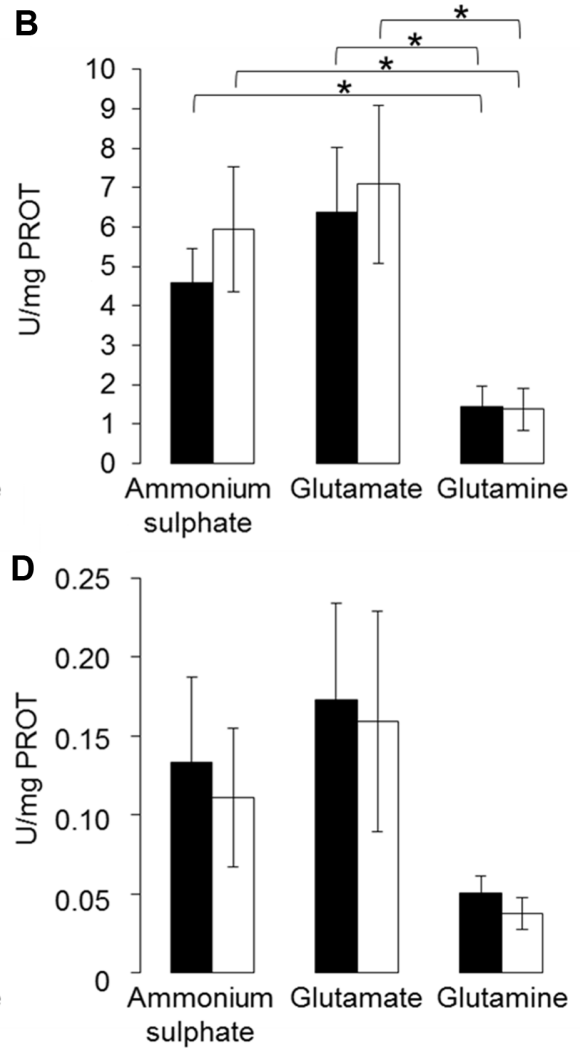

Fig. 7. Activities of enzymes of central nitrogen metabolism, tested on the soluble protein fraction of CEN.PK C (black columns) and CEN.PK TPI-GLT1 (white columns), cultured in media with the indicated nitrogen source. (A) Glutamate synthase activity. (B) Glutamine synthetase activity. (C) Glutamate dehydrogenase 2 activity. (D) Glutamate dehydrogenase activity. Nitrogen sources were supplied to release $30.6 \mathrm{mM}$ of ammonium.

Values represent the average and the standard deviation of three independent experiments $\left({ }^{*} p \leq 0.01\right.$; \#p $\leq 0.05$; Student's $t$-test).

fermentation processes [1, 17]. Indeed, the replacement of petrochemistry-based transport fuels and bulk chemicals by yeast industrial biotechnology requires cost-effective fermentation processes, where yields of substrate conversion into product must approach the maximal theoretical values.

Only very few manuscripts looking over the engineering of the central nitrogen metabolism of S. cerevisiae have been published [25, 27, 32, 38]. In all these works, the redox intracellular status was unbalanced to decrease glycerol levels and to increase the ethanol production in aerobic or anaerobic batch, using ammonium sulfate as a sole nitrogen source. To reach this purpose, GLT1 has always been overexpressed, with a contemporary deletion of GPD1 and GPD2 (encoding for glycerol-3-phosphate dehydrogenases) [38], or with an additional GDH1 deletion and GLN1 overexpression [25, 27, 32]. Improvements in terms of ethanol yield and production have been reached in some cases $[27,38]$, but the ethanol productivity resulted negatively affected by the reduced specific growth rate of the strains.

To focus the attention on the GLT1 contribution in the CNM, we analyzed the effects of GLT1 modulation by growing wild-type, glt1 1 , and GLT1 overexpressing strains under different physiological conditions. To the best of our knowledge, this work is the first describing the physiology of $S$. cerevisiae strains exclusively modulating the GLT1 expression.

At the cellular level, almost all nitrogen sources are catabolized to glutamate, which plays a key role in the direct biosynthesis of all the others amino acids, except for asparagine and tryptophan, which are synthesized starting from glutamine [21]. In the experiments, high or low ammonium sulfate (as control), glutamate, or glutamine concentrations have been used. Furthermore, a high glucose concentration $(150 \mathrm{~g} / \mathrm{l})$ has been chosen with a double purpose; on the one hand to balance and support the high nitrogen supply and on the other hand to simulate a condition often applied for industrial yeast fermentations. 
It is important to underline that such concentration is undoubtedly high for typical laboratory scale experiments and it could furthermore cause glucose repression on different genes, including those of the CNM. For example, an implication of Snif1p kinase, one of the major cytoplasmic glucose sensors, has been discovered in the regulation cascade of GLT1 expression [34]. High glucose concentrations can inactivate this kinase [18], which, in turn, prevents Gln3p nuclear accumulation [2]. Consequently, it is important to consider that in the presented experimental setting the expression of genes controlled by this transcription factor, as GDH1 [31], GDH2, and GLN1 [23], could be affected by such high glucose concentration.

Data obtained indicate that a different GLT1 background does not interfere with the growth properties of the strains when cultivated on ammonia, glutamate, or glutamine as nitrogen sources, even if in the overexpressing strain the Glt1p activity is greatly enhanced compared with the control strain. However, as an important exception, the GLT1 overexpressing strain was less viable only in the presence of glutamine as nitrogen source. These data taken together confirm and highlight the robustness of the CNM of yeast against internal perturbation, and at the same time its plasticity with respect to the environment. Indeed, a strong modulation of yeast growth is obtained by using different nitrogen sources. The highest biomass productions are obtained by formulating media with glutamine, whereas glutamate confers higher cellular viability and robustness compared with the other media.

Considering the strong metabolic dependency of the two amino acids at the CNM node (see Fig. 1), a difference between glutamate and glutamine (as shown in Fig. 6) was not trivial to anticipate. This difference suggests a proactive role of glutamate to guarantee a generally higher cellular robustness. Nevertheless, data shown in Fig. 6 (Panels D-F) clearly indicate that with this nitrogen source, two distinct yeast subpopulations, which react to the external stressor in a very different way, appear. Moreover, one subpopulation can easily prevent the formation of ROS species. In this respect, GLT1 deletion seems to have a negative consequence, since it doubles (from $\sim 24 \%$ to $\sim 40 \%$ ) the fraction of cells accumulating ROS. Diversely, there is no variation in the abundance of this subpopulation when GLT1 is overexpressed. Currently, we do not have speculations for the interpretation of the data. However, it can be anticipated that understanding these aspects might foster the design of much robust yeast cell factories. Finally, when cells are supported with ammonium sulfatebased medium (the nitrogen source mainly used at laboratory level), the lowest optical density associated to the lowest viability is observed. This needs to be taken into account when results obtained at laboratory scale are important for speculating about industrial scale-up, and opens further consideration on media formulation.

\section{Acknowledgments}

P.B., G.F., and D.P. acknowledge the support by FAR (Fondo di Ateneo per la Ricerca) of the University of Milano-Bicocca; D.P. and G.F. acknowledge the partial support of the SYSBIO - Centre of Systems Biology (SysBioNet, Italian Roadmap for ESFRI Research Infrastructure). M.B. acknowledges the $\mathrm{PhD}$ fellowship of the University of Milano Bicocca.

\section{References}

1. Abdel-Rahman MA, Tashiro Y, Sonomoto K. 2013. Recent advances in lactic acid production by microbial fermentation processes. Biotechnol. Adv. 31: 877-902.

2. Bertram PG, Choi JH, Carvalho J, Chan TF, Ai W, Zheng XF. 2002. Convergence of TOR-nitrogen and Snf1-glucose signaling pathways onto Gln3. Mol. Cell. Biol. 22: 1246-1252.

3. Bradford MM. 1976. A rapid and sensitive method for the quantitation of microgram quantities of protein utilizing the principle of protein-dye binding. Anal. Biochem. 72: 248-254.

4. Branduardi P, Porro D. 2012. Yeasts in Biotechnology, pp. 347-370. In Feldmann $\mathrm{H}$ (ed). Yeast: Molecular and Cell Biology. John Wiley \& Sons Inc., Germany.

5. Branduardi P, Fossati T, Sauer M, Pagani R, Mattanovich D, Porro D. 2007. Biosynthesis of vitamin C by yeast leads to increased stress resistance. PLoS One 2: e1092.

6. Cao J, Barbosa JM, Singh NK, Locy RD. 2013. GABA shunt mediates thermotolerance in Saccharomyces cerevisiae by reducing reactive oxygen production. Yeast 30: 129-144.

7. Cogoni C, Valenzuela L, González-Halphen D, Olivera H, Macino G, Ballario P, González A. 1995. Saccharomyces cerevisiae has a single glutamate synthase gene coding for a plant-like high-molecular-weight polypeptide. J. Bacteriol. 177: 792-798.

8. Coleman ST, Fang TK, Rovinsky SA, Turano FJ, MoyeRowley WS. 2001. Expression of a glutamate decarboxylase homologue is required for normal oxidative stress tolerance in Saccharomyces cerevisiae. J. Biol. Chem. 276: 244-250.

9. Crespo JL, Powers T, Fowler B, Hall MN. 2002. The TORcontrolled transcription activators GLN3, RTG1, and RTG3 are regulated in response to intracellular levels of glutamine. Proc. Natl. Acad. Sci. USA 99: 6784-6789.

10. DeLuna A, Avendano A, Riego L, Gonzalez A. 2001. NADPglutamate dehydrogenase isoenzymes of Saccharomyces 
cerevisiae. Purification, kinetic properties, and physiological roles. J. Biol. Chem. 276: 43775-43783.

11. Edwards AN, Patterson-Fortin LM, Vakulskas CA, Mercante JW, Potrykus K, Vinella D, et al. 2011. Circuitry linking the Csr and stringent response global regulatory systems. Mol. Microbiol. 80: 1561-1580.

12. Gietz RD, Woods RA. 2002. Transformation of yeast by lithium acetate/single-stranded carrier DNA/polyethylene glycol method. Methods Enzymol. 350: 87-96.

13. Guillamón JM, van Riel NA, Giuseppin ML, Verrips CT. 2001. The glutamate synthase (GOGAT) of Saccharomyces cerevisiae plays an important role in central nitrogen metabolism. FEMS Yeast Res. 1: 169-175.

14. Hohenblum H, Borth N, Mattanovich D. 2003. Assessing viability and cell-associated product of recombinant protein producing Pichia pastoris with flow cytometry. J. Biotechnol. 102: 281-290.

15. Holmes AR, Collings A, Farnden KJ, Shepherd MG. 1989. Ammonium assimilation by Candida albicans and other yeasts: evidence for activity of glutamate synthase. J. Gen. Microbiol. 135: 1423-1430.

16. Hong KK, Nielsen J. 2012. Metabolic engineering of Saccharomyces cerevisiae: a key cell factory platform for future biorefineries. Cell. Mol. Life Sci. 69: 2671-2690.

17. Huo YX, Cho KM, Rivera JG, Monte E, Shen CR, Yan Y, Liao JC. 2011. Conversion of proteins into biofuels by engineering nitrogen flux. Nat. Biotechnol. 29: 346-351.

18. Kayikci Ö, Nielsen J. 2015. Glucose repression in Saccharomyces cerevisiae. FEMS Yeast Res. 15. DOI: 10.1093/femsyr/fov068.

19. Kim YM, Hong SJ, Billiar TR, Simmons RL. 1996. Counterprotective effect of erythrocytes in experimental bacterial peritonitis is due to scavenging of nitric oxide and reactive oxygen intermediates. Infect. Immun. 64: 3074-3080.

20. Kingdon HS, Hubbard JS, Stadtman ER. 1968. Regulation of glutamine synthetase. XI. The nature and implications of a lag phase in the Escherichia coli glutamine synthetase reaction. Biochemistry 7: 2136-2142.

21. Ljungdahl PO, Daignan-Fornier B. 2012. Regulation of amino acid, nucleotide, and phosphate metabolism in Saccharomyces cerevisiae. Genetics 190: 885-929.

22. Madeo F, Fröhlich E, Ligr M, Grey M, Sigrist SJ, Wolf DH, Fröhlich KU. 1999. Oxygen stress: a regulator of apoptosis in yeast. J. Cell Biol. 145: 757-767.

23. Magasanik B, Kaiser CA. 2002. Nitrogen regulation in Saccharomyces cerevisiae. Gene 290: 1-18.

24. Miller JS, Quarles JM. 1990. Flow cytometric identification of microorganisms by dual staining with FITC and PI. Cytometry 11: 667-675.

25. Moreira dos Santos M, Thygesen G, Kötter P, Olsson L, Nielsen J. 2003. Aerobic physiology of redox-engineered Saccharomyces cerevisiae strains modified in the ammonium assimilation for increased NADPH availability. FEMS Yeast Res. 4: 59-68.
26. Murthy GS, Johnston DB, Rausch KD, Tumbleson ME, Singh V. 2012. A simultaneous saccharification and fermentation model for dynamic growth environments. Bioprocess Biosyst. Eng. 35: 519-534.

27. Nissen TL, Kielland-Brandt MC, Nielsen J, Villadsen J. 2000. Optimization of ethanol production in Saccharomyces cerevisiae by metabolic engineering of the ammonium assimilation. Metab. Eng. 2: 69-77.

28. Penninckx MJ. 2002. An overview on glutathione in Saccharomyces versus non-conventional yeasts. FEMS Yeast Res. 2: 295-305.

29. Perrone GG, Tan SX, Dawes IW. 2008. Reactive oxygen species and yeast apoptosis. Biochim. Biophys. Acta 1783: 1354-1368.

30. Porro D, Gasser B, Fossati T, Maurer M, Branduardi P, Sauer M, Mattanovich D. 2011. Production of recombinant proteins and metabolites in yeasts: when are these systems better than bacterial production systems? Appl. Microbiol. Biotechnol. 89: 939-948.

31. Riego L, Avendaño A, DeLuna A, Rodríguez E, González A. 2002. GDH1 expression is regulated by GLN3, GCN4, and HAP4 under respiratory growth. Biochem. Biophys. Res. Commun. 293: 79-85.

32. Roca C, Nielsen J, Olsson L. 2003. Metabolic engineering of ammonium assimilation in xylose-fermenting Saccharomyces cerevisiae improves ethanol production. Appl. Environ. Microbiol. 69: $4732-4736$.

33. Soberón M, González A. 1987. Physiological role of glutaminase activity in Saccharomyces cerevisiae. J. Gen. Microbiol. 133: 1-8.

34. Usaite R, Wohlschlegel J, Venable JD, Park SK, Nielsen J, Olsson L, Yates III JR. 2008. Characterization of global yeast quantitative proteome data generated from the wild-type and glucose repression Saccharomyces cerevisiae strains: the comparison of two quantitative methods. J. Proteome Res. 7: 266-275.

35. Valenzuela L, Ballario P, Aranda C, Filetici P, González A. 1998. Regulation of expression of GLT1, the gene encoding glutamate synthase in Saccharomyces cerevisiae. J. Bacteriol. 180: 3533-3540.

36. Verduyn C, Postma E, Scheffers WA, Van Dijken JP. 1992. Effect of benzoic acid on metabolic fluxes in yeasts: a continuous-culture study on the regulation of respiration and alcoholic fermentation. Yeast 8: 501-517.

37. Wach A, Brachat A, Pöhlmann R, Philippsen P. 1994. New heterologous modules for classical or PCR-based gene disruptions in Saccharomyces cerevisiae. Yeast 10: 1793-1808.

38. Wang J, Liu W, Ding W, Zhang G, Liu J. 2013. Increasing ethanol titer and yield in a gpd1 $1 \Delta p d 2 \Delta$ strain by simultaneous overexpression of GLT1 and STL1 in Saccharomyces cerevisiae. Biotechnol. Lett. 35: 1859-1864.

39. Zhao X, Zou H, Fu J, Chen J, Zhou J, Du G. 2013. Nitrogen regulation involved in the accumulation of urea in Saccharomyces cerevisiae. Yeast 30: 437-447. 